\title{
Development of a Pulsed High Current Facility for Accelerating Metal Foils for Hydrodynamic Materials Testing
}

\author{
N. Graneau* and K. Omar \\ AWE, Aldermaston, Berkshire, RG7 4PR, UK
}

\begin{abstract}
A new pulsed power machine to be called AMPERE is currently under development at AWE. Its initial use will be as a metallic foil accelerator for the specific purpose of applying large impulses ( $>300 \mathrm{~Pa}$ s) and pressures $(>450 \mathrm{G} \mathrm{Pa})$ to a target for material property testing. The critical circuit features are described and a computer model has been written to predict the expected machine performance. Future uses of this facility for driving further hydrodynamic experimentation are also discussed.
\end{abstract}

PACS numbers: 84.70.+p, 84.30.Ng, 62.50.Ef

\section{Introduction}

The initial aim of this development programme is to create a Magnetic Foil Flyer. High velocity foils and plates impacting on a target surface are an effective method of applying a specific impulse to a significant surface area of a structure and generating planer shock waves. We aim to accelerate a several hundred $\mathrm{cm}^{2}$ foil of several hundred $\mu \mathrm{m}$ thickness, up to several hundred $\mathrm{m} / \mathrm{s}$. The foil will be driven by electrodynamic forces generated during the discharge of a $120 \mathrm{~kJ}(40 \mathrm{kV})$ capacitor bank, configured to produce maximum currents of around $600 \mathrm{kA}$. For the purpose of accelerating foils weighing between 3-43 g over a distance of around $3 \mathrm{~mm}$, it is desirable to generate current pulses with a width of $10-20 \mu \mathrm{s}$ and rise times of 5-10 $\mu \mathrm{s}$. By appropriate choice of capacitors and switch, these requirements can be achieved without any further pulse sharpening circuit components. The pulsed current flows through the foil which is accelerated by the electromagnetic force of repulsion between it and a nearby high inertial mass parallel return conductor.

Proposed future applications include other electrodynamically driven systems for the purpose of hydrodynamic equation of state testing of uniform and composite materials without using high explosives. Sequential switching techniques will allow pulse shaping for conventional isentropic compression experiments (ICE), but also untried techniques for large controlled force production involving arc discharges in water [1] or other media will be attempted. A further set of experiments is envisioned which will utilize the magnetic hoop stress in a rapidly

\footnotetext{
* corresponding author; e-mail: neal.graneau@awe.co.uk
}

pulsed solenoid load to apply outward radial shocks to rings or cylindrical structures [2].

\section{Critical circuit components}

\subsection{Foil flyer strip-line}

The proposed structure of the foil flyer strip-line (FFSL) is shown in Fig. 1. The first priority of the design was to ensure that the foil flyer (FF) is mounted in close proximity to the return conductor. A $2 \mathrm{~mm}$ initial separation between the foil and bottom current return conductor will be maintained by an insulating Mylar sheet. The copper conductors on either end of the foil flyer will be held down firmly as they will also be subject to forces of up to $300 \mathrm{kN}$.

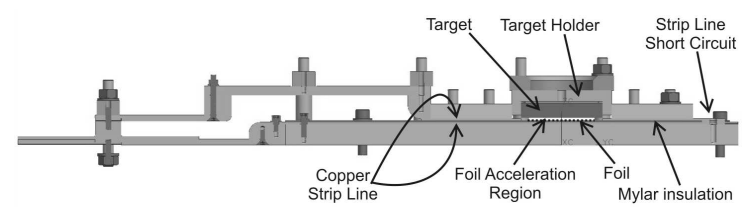

Fig. 1. Centre section through FFSL, highlighting the foil acceleration region.

The simplified side view of the circuit that we analyzed is shown in Fig. 2. All the conductors have the same thickness $(250 \mu \mathrm{m})$ and the length of the strip line on either end of the foil is $20 \mathrm{~cm}$. In this example, the strip line is $9 \mathrm{~cm}$ wide and the square $\mathrm{FF}$ is thus $9 \times 9 \mathrm{~cm}^{2}$ in size. We separately calculated the force components on the foil due to the bottom (return) conductor and the two non-foil sections of the top line. Results including the total force acting on the foil are displayed in Fig. 2. 


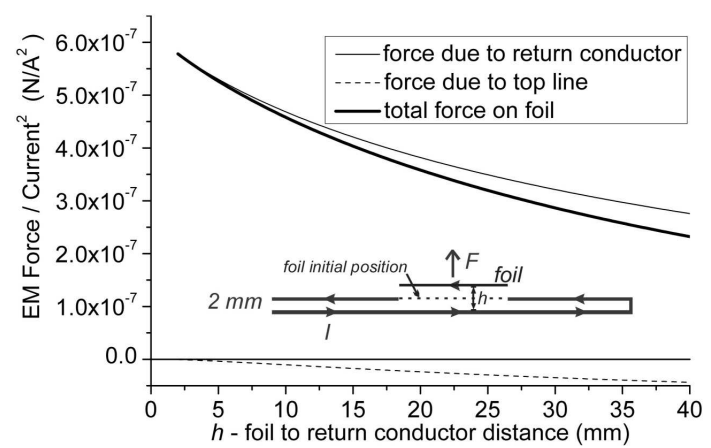

Fig. 2. Calculated electrodynamic force acting on the foil flyer.

\subsection{Exploding foil dynamic resistor and parallel damping resistor}

In order to reduce capacitor exposure to excessive reverse voltage, a dynamic resistance foil (50 $\mu \mathrm{m}$ thick) is included, designed to explode and go open circuit near the first current zero. This still allows a high current to flow as the exploding foil will have a fairly low resistivity during the first current maximum. A $1.6 \Omega$ damping resistor will be placed in parallel with the foil to reduce any voltage transients due to rapid current chopping. Re-strike will be avoided by embedding the foil in sand onto which the exploded liquid metal particles can condense [3].

\section{Modelling the foil flyer performance}

Inclusion of the exploding foil dynamic resistor (EFDR) introduces a dynamic resistance which changes significantly during the current pulse. To a lesser extent, the FF itself is thin enough that it may heat up to about $20-30 \%$ of its explosion temperature and will display dynamic resistance. Both of these effects are modelled. Inductance increase due to switching arc constriction and foil motion are considered negligible and therefore ignored. The IDL (Interactive Data Language) computer code therefore analyses six major features of the foil flyer system.

1. Circuit current and capacitor voltage.

2. Foil flyer - energy absorption and resistance.

3. Exploding foil dynamic resistor - energy absorption and resistance.

4. Electromagnetic and air pressure forces acting on the FF.

5. Kinematics (acceleration, velocity and position) of the FF.

6. Impulse imparted to target.
The electrical, thermal and mechanical properties of the circuit are monitored during a series of time steps. At each iteration, the amount of thermal energy deposited in the EFDR and the FF during that interval is calculated by knowledge of the current and the resistivity of the respective foil. We can relate the resistivity $(\mu \Omega \mathrm{cm})$ of each foil to the accumulated internal energy density $(\mathrm{kJ} / \mathrm{g})$ by referring to published experimental data [4]. At each time step, the total circuit resistance is re-assessed. Given a relationship between (EM force/current ${ }^{2}$ ) vs. (foil height) from Fig. 2, as well as the calculated current pulse shape, we can quantify the acceleration, velocity and position of the FF as well as estimating the mechanical impulse imparted to a target placed at known distance from the initial foil position. When approaching a planar target we assume that the compression happens fast enough that an adiabatic air compression model is appropriate.

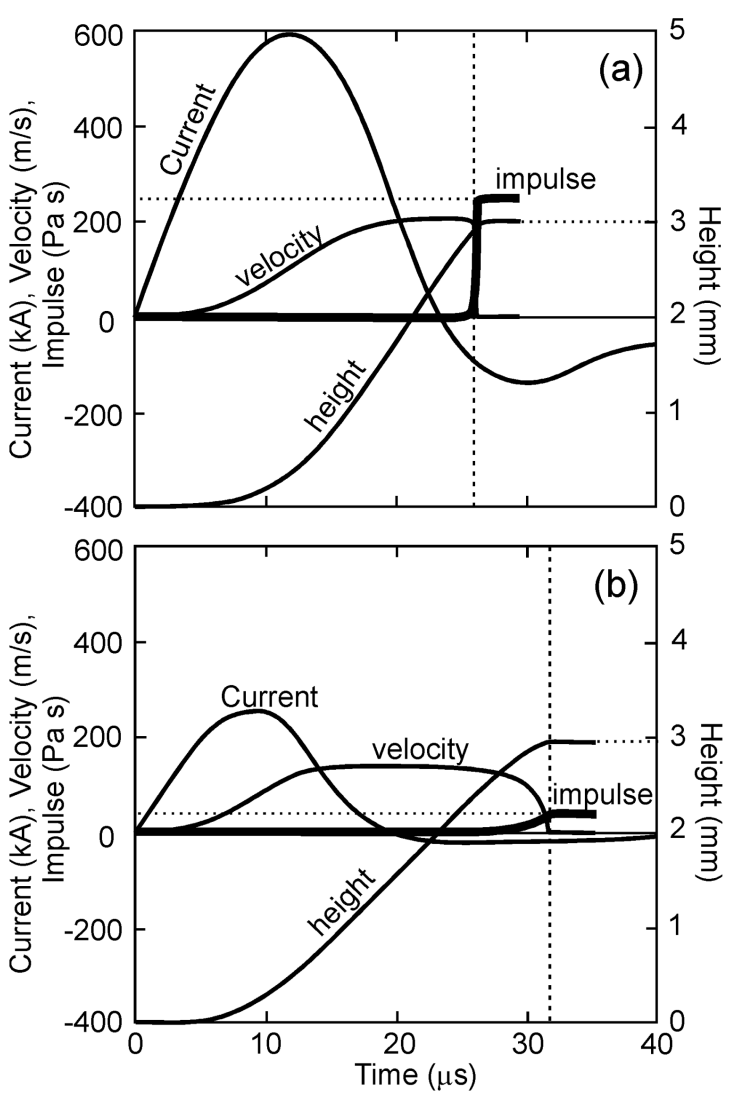

Fig. 3. Typical calculations of FF velocity and impulse delivered to target. Common parameters: foil flyer $=9 \times 9 \mathrm{~cm}^{2}$, target height $=3 \mathrm{~mm}$, EFDR foil $30 \mathrm{~cm}$ long, $50 \mu \mathrm{m}$ thick, $C=150 \mu \mathrm{F}$. (a) INPUTS: $\mathrm{FF}$ foil thickness $=500 \mu \mathrm{m}$, total inductance $=400 \mathrm{nH}$, EFDR foil width $=16 \mathrm{~cm}, V_{0}=36 \mathrm{kV}$; OUTPUTS: velocity $(\max )=205 \mathrm{~m} / \mathrm{s}$, impulse on target $=247 \mathrm{~Pa} \mathrm{~s}$, (b) INPUTS: FF foil thickness $=100 \mu \mathrm{m}$, total inductance $=200 \mathrm{nH}$, EFDR foil width $=6 \mathrm{~cm}, V_{0}=15 \mathrm{kV}$; OUTPUTS: velocity $(\max )=139 \mathrm{~m} / \mathrm{s}$; Impulse on target $=38 \mathrm{~Pa} \mathrm{~s}$. 
Our primary modelling aim was to ensure that under various circuit modifications, we could predict the acceleration of an aluminium $\mathrm{FF}$ to a specified velocity with which to impart a predictable impulse on a target. We have estimated that the circuit resistance excluding the two foils is $3 \mathrm{~m} \Omega$ and in all cases the initial separation between the FF and lower conductor is $2 \mathrm{~mm}$. The computer model investigated the FF performance over a range of circuit inductances.

The four quantities that were varied in the two sample calculations shown here were the thickness of the FF foil, circuit inductance, width of the exploding EFDR foil and the initial voltage on the capacitor bank, $V_{0}$. In both cases, the aluminium FF is $9 \times 9 \mathrm{~cm}^{2}$ and the aluminium EFDR exploding foil is $50 \mu \mathrm{m}$ thick and $30 \mathrm{~cm}$ long. Results from the two calculations are depicted in Fig. 3. This model will allow us to select the optimum circuit conditions for a given impulse loading requirement on a target material.

\section{Conclusions}

A comprehensive modeling exercise has been undertaken in order to design a high current pulsed power facility which can accelerate metal foils up to sufficient velocities for planned materials testing. The computer calculations have revealed that we will be able to achieve our initial minimum specification of launching foils of size $(10 \mathrm{~cm} \times 20 \mathrm{~cm} \times 400 \mu \mathrm{m})$ up to velocities of more than $300 \mathrm{~m} / \mathrm{s}$ within a distance of $3 \mathrm{~mm}$, allowing us to impart a specific areal impulse to a target material of at least $300 \mathrm{~Pa}$ s, implying a peak pressure of $470 \mathrm{GPa}$ (4.7 Mbar). Future modeling will hopefully reveal more optimised geometries for FF load (FFSL) which will ensure a more uniform force distribution across the foil surface.

AMPERE will also be configured to drive other loads. Some experiments will utilize the electromagnetic hoop stress in a solenoid which can be pulsed to apply radial shocks to tubular structures. We also aim to create high current arcs in semi-enclosed water cavities as a means of producing large ramped pressure waves for isentropic compression measurements and other hydrodynamic materials testing.

\section{References}

[1] H. Bluhm, Pulsed Power Systems, Springer 2006.

[2] W.H. Gourdin, J. Appl. Phys 65, 411 (1989).

[3] J.C. Bueck, R.E. Reinovsky, 5th IEEE Int. Pulsed Power Conf., Eds. M.F. Rose, P.J. Turchi, 1985, p. 287.

[4] W.F. McCullough, in Ref. [3], p. 291. 